\title{
A novel multiple-trauma CT-scanning protocol using patient repositioning may increase risks of iatrogenic injuries
}

\author{
L. M. Benneker • H. M. Bonel • M. A. Zumstein • \\ A. K. Exadaktylos
}

Received: 24 November 2006 / Accepted: 20 December 2006/ Published online: 15 February 2007

(C) Am Soc Emergency Radiol 2007

\section{Dear Editor,}

Hoppe et al. [1] from the Department of Radiology at Inselspital Bern reported the potential benefits of a novel multiple-trauma CT-scanning protocol using patient repositioning. In the study of our distinguished fellow radiologists, a novel protocol with patient rotation from headfirst and arms at the side with arms raised for scanning the chest, abdomen and pelvis was compared to a headfirst CT protocol without repositioning.

The authors suggest that during a whole body scan, repositioning of the patient to reduce artefacts caused by arms or installations improves image quality. The authors conclude that this novel multiple-trauma CT-scanning protocol with patient repositioning achieves a higher image quality with significantly fewer artefacts than without repositioning. The authors suggest the use of this technique in patients without clinically suspected fractures and/or dislocation of the upper extremities [2].

L. M. Benneker $(\bowtie) \cdot$ M. A. Zumstein

Department of Orthopedic Surgery,

University Hospital of Berne,

3010 Bern, Switzerland

e-mail: lorin.benneker@insel.ch

H. M. Bonel

Department for Radiology,

University Hospital of Berne,

Bern, Switzerland

\section{A. K. Exadaktylos}

Department for Emergency Medicine,

University Hospital of Berne,

Bern, Switzerland
Although we agree that this kind of positioning is beneficial for better imaging, we would like to question the use of this technique in intubated, sedated and paralysed patients due to a recent incident we had.

Case A 39-year-old female patient was referred to our unit after a fall from a height. Due to a closed brain injury, she was intubated, ventilated, and muscle relaxants were given. The rescue team reported voluntary movement of all extremities before intubation. Bony injuries were ruled out clinically according the ATLS primary survey algorithm and using a low dose full body ap/lat X-ray (Lodox Systems, Statscan, Benmore, SA; Fig. 1).

Patient was referred to CT for further imaging. Again "CT scout" and cervical spine reconstructions showed neither fractures nor dislocations before bringing the arms in the above head position like described by our colleagues (Fig. 2).

After performing thoraco-abdominal imaging, patients arms were brought back at side. No obvious problems were noted during this procedure. Patient was transferred to ICU, where, after extubation, an anterior glenohumeral dislocation and consecutive plexus injury has been diagnosed (Fig. 3), which occurred either initially at trauma or was caused at iatrogenic luxation during repositioning or at the following manipulation (adduction) of the antero-inferiorly dislocated humerus. The dislocation was missed on CT chest scout and axial images (Figs. 4 and 5) and clinically due to the effect of muscle relaxants, which cause the musculoskeletal apparatus to be extremely flaccid and difficult to assess. Relocation was unproblematic, but due 


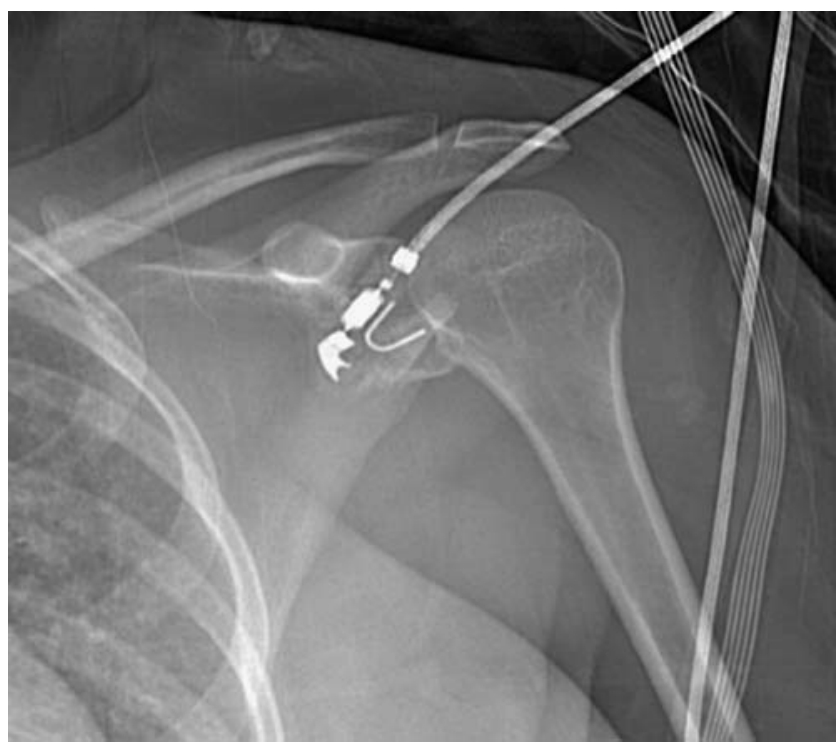

Fig. 1 Shoulder section of low-dose full-body ap radiograph (lodox), note the correct articulation

to a probable traction injury of the plexus brachialis, paresis of the left arm remained. Retrospective analysis of the first CT scans for the cervical spine reveal already a severe hematoma ventral of $\mathrm{M}$. subscapularis. This suggests that instability and probably also the plexus injury had a traumatic cause - whether there occurred additional iatrogenic traction injury remains difficult to assess.

We report therefore the first complication of this suggested technique. The repositioning manoeuvre mimics

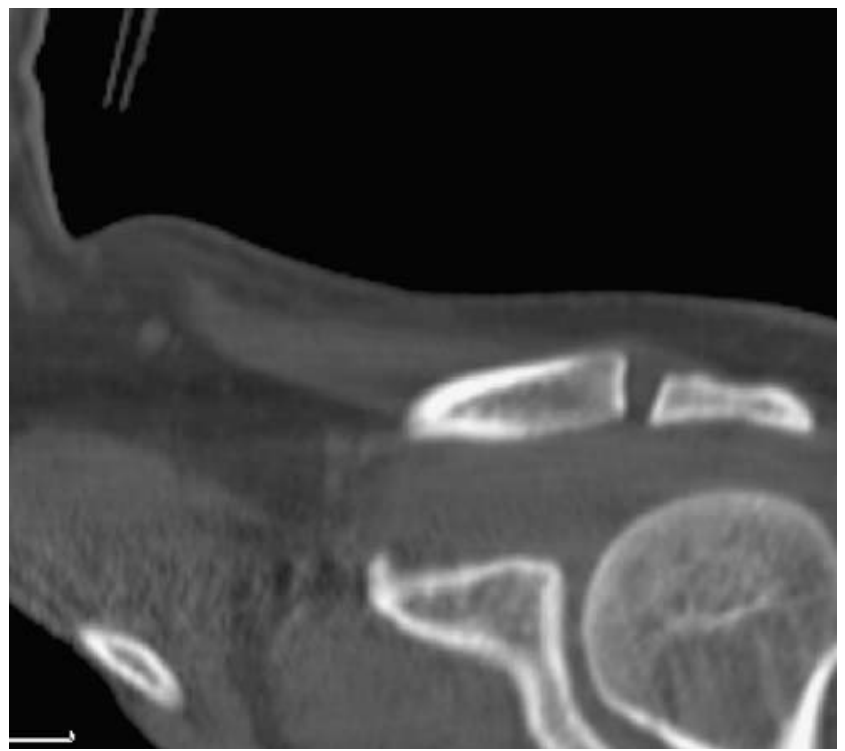

Fig. 2 Coronary reconstruction of glenohumeral joint from the cervical spine/cranium CT protocol (before repositioning), head of humerus still in place

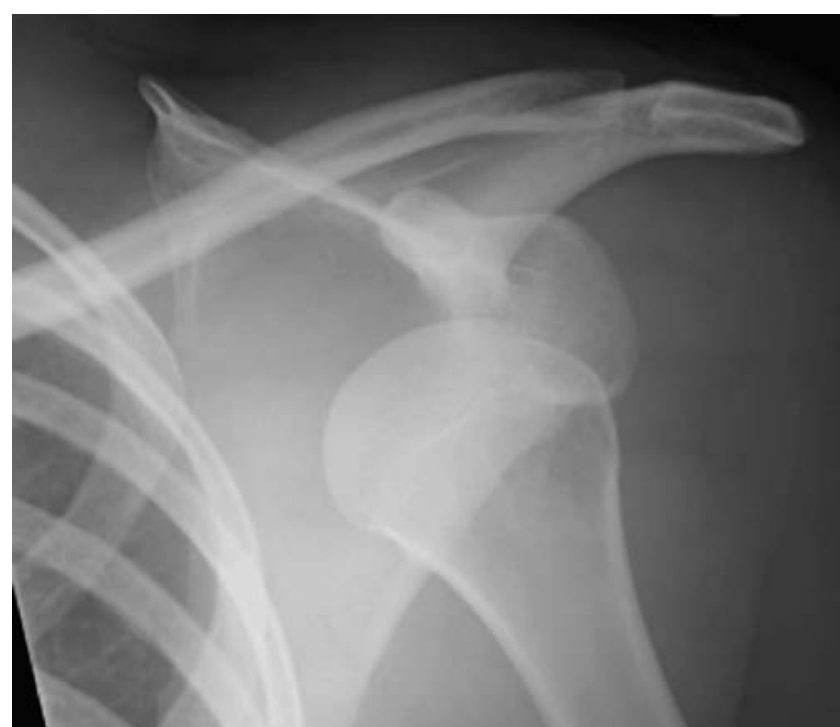

Fig. 3 Documentation of the anterior glenohumeral dislocation

the classic apprehension test for anterior glenohumeral instability and with a hardened vacuum mattress as a dorsal fulcrum. Anterior dislocation in the intubated patient is possible with pre-existing or traumatic instability. The use of a repositioning CT protocol in ventilated and paralysed patients requires special attention to the shoulder region to avoid iatrogenic injuries such as anterior shoulder dislocation.

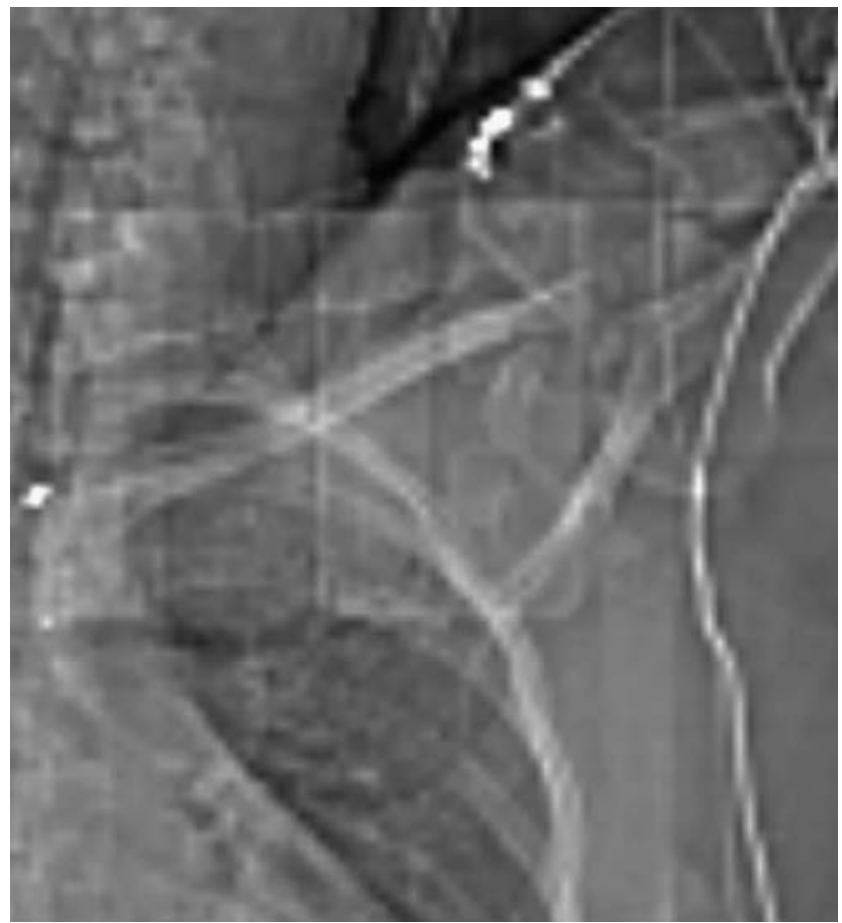

Fig. 4 Retrospective evaluation of CT scout for the chest, abdomen and pelvis scan shows antero-inferior shoulder dislocation after repositioning 


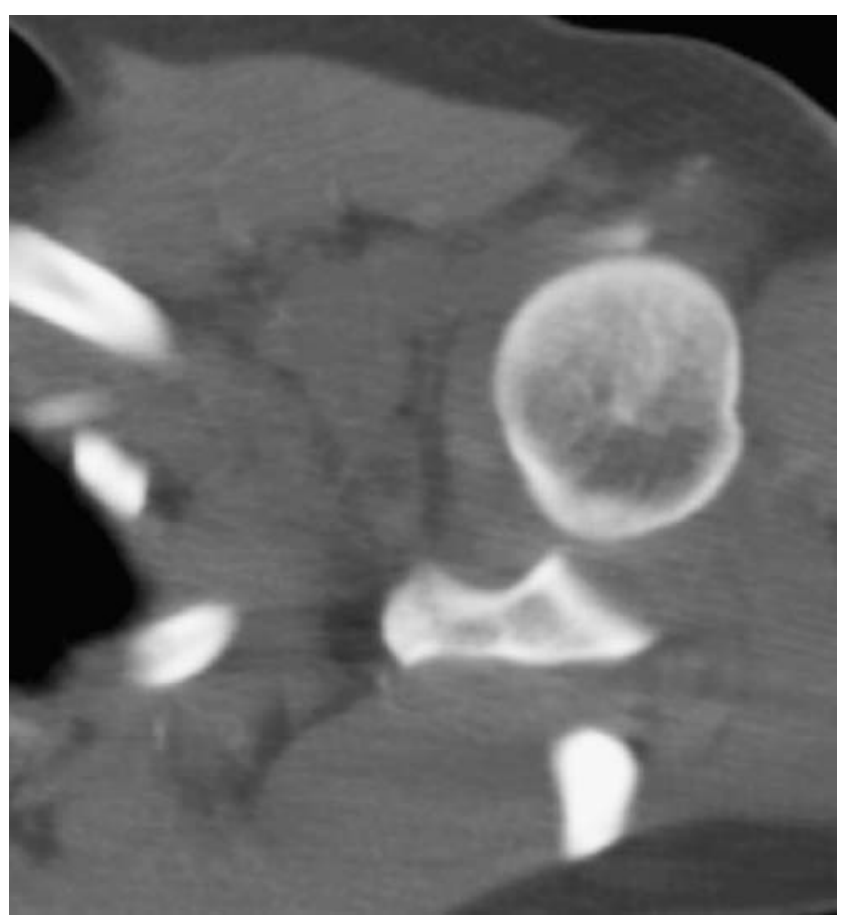

Fig. 5 Retrospective evaluation of axial images for the chest, abdomen and pelvis CT scan shows antero-inferior shoulder dislocation after repositioning

\section{References}

1. Hoppe H, Vock P, Bonel HM, Ozdoba C, Gralla J (2006) A novel multiple-trauma CT-scanning protocol using patient repositioning. Emerg Radiol 13(3):123-128

2. Linsenmaier U, Krotz M, Hauser H, Rock C, Rieger J, Bohndorf K, Pfeifer KJ, Reiser M (2002) Whole-body computed tomography in polytrauma: techniques and management. Eur Radiol 12(7):17281740 (Epub 2001 Dec 13) 